\title{
A Study of Violet-pigmented Micrococci. Yellow-pigmented Mutants of Staphylococcus flavocyaneus
}

\author{
By S. ROSYPAL, M. KOCUR AND K. HOĎÁK \\ Department of Microbiology, Faculty of Sciences, \\ University of J. E. Purkynĕ, Brno, Czechoslovakia
}

(Received 13 April 1962)

\begin{abstract}
SUMMARY
Staphylococcus flavocyaneus produces at least two pigments on glucose yeast-extract agar; a violet diffusible pigment and a yellow non-diffusing pigment. On some media the production of violet diffusible pigment is inhibited. In broth cultures of this organism a mutation takes place giving rise to yellow-pigmented mutants which have lost the ability of producing the violet pigment. A considerable accumulation of these mutants occurs in cultures on both solid and liquid media. No reversion to the violetpigmented state or other change in pigmentation in the mutants has been observed.

The yellow-pigmented mutants do not differ from strains of Micrococcus luteus with the exception of some characteristics which are variable within that species. This supports the conclusions of Kocur \& Martinec (1963) that Staphylococcus flavocyaneus Knaysi should be regarded as a strain of Micrococcus luteus (Schroeter) Cohn.
\end{abstract}

\section{INTRODUCTION}

Kocur \& Martinec (1963) concluded that the violet-pigmented cocci named Staphylococcus flavocyaneus Knaysi, Micrococcus cyaneus (Schroeter) Cohn, and $M$. polychromus Makarova are identical with $\boldsymbol{M}$. luteus (Schroeter) Cohn. Knaysi (1942) observed that one of these cocci described under the name $S$. flavocyaneus dissociated spontaneously into a lemon-yellow strain. Under our cultural conditions on the glucose yeast-extract agar $\boldsymbol{S}$. flavocyaneus produced, according to Knaysi's observation (1942), two pigments, one violet and diffusible, the other yellow and non-diffusing. In preliminary experiments we found that the accumulation of yellow-pigmented variants occurred in the broth cultures of $\boldsymbol{S}$. flavocyaneus even if we initiated the cultures with very small inocula that did not contain the yellow variants. In addition the proportion of yellow-pigmented variants varied signicantly between similar broth cultures of this organism. According to these findings, which will be described in detail in a subsequent paper, we consider the yellow variants of $\boldsymbol{S}$. flavocyaneus to be spontaneous mutants. Here we describe the population changes in broth cultures of $S$. flavocyaneus due to the accumulation of the yellow-pigmented mutants, and the taxonomic significance of this in the classification of $\boldsymbol{S}$. flavocyaneus will be considered. 


\section{METHODS}

Most of the observations described in this article were made with the strain BS 852 designated by us as Staphylococcus flavocyaneus and received from Professor C. B. van Niel. Other experiments were made with the original strain of Knaysi S. flavocyaneus (NCTC 7011), and with strains BS 851 and BS 856 (see Kocur \& Martinec, 1963). We found the same phenomenon in Knaysi's type strain. The strains given in Table 3 come from our collection.

Gurr's nutrient broth was used for the studies of the accumulation of mutants. Viable counts were made by plating $0.1 \mathrm{ml}$. of appropriate dilutions on glucose yeast-extract agar (g./l. distilled water: yeast extract, 5; peptone, 5; glucose, 10; agar, 10; adjusted to $\mathrm{pH} \mathrm{7 \cdot 2).} \mathrm{The} \mathrm{following} \mathrm{media} \mathrm{were} \mathrm{also} \mathrm{used:} \mathrm{Difco} \mathrm{nutrient}$ agar; nutrient agar with gelatin (Difco nutrient agar, $900 \mathrm{ml} . ; 10 \%(\mathrm{w} / \mathrm{v})$ gelatin, $10 \mathrm{ml}$; ; adjusted to $\mathrm{pH} 7 \cdot 2)$; Difco nutrient agar with $2 \%(\mathrm{w} / \mathrm{v})$ soluble starch (adjusted to $\mathrm{pH} 7 \cdot 2$ ); yeast-extract agar with phenylalanine (g./l. distilled water: yeast extract, 3; DL-phenylalanine, $2 ; \mathrm{Na}_{2} \mathrm{HPO}_{4}, 1 ; \mathrm{NaCl}, 5$; agar, 10); glucose yeast-extract agar with beef extract (g./1. distilled water; yeast extract, 3 ; beef extract, 3; glucose, $20 ; \mathrm{CaCO}_{3}, 20$; agar, 20).

Serial transfer of Bs $\mathbf{8 5 2}$ was carried out as follows: Erlenmeyer's flasks, each containing $10 \mathrm{ml}$. broth, were inoculated with $878 \pm\left(t s_{\bar{x}}=65, P=0.05\right)$ cells of strain Bs 852. Control platings showed that there were no yellow-pigmented mutants in the inocula. The flasks were incubated for $96 \mathrm{hr}$. at $30^{\circ}$ and $0 \cdot 1 \mathrm{ml}$. was transferred into flasks containing fresh broth. Similar transfers were repeated ten times, but at $48 \mathrm{hr}$. intervals. After the second, fourth, sixth, eighth and tenth transfers platings of suitable dilutions were made on glucose yeast-extract agar and incubated at $30^{\circ}$ for $72 \mathrm{hr}$. to determine the percentage of yellow-pigmented mutants. The same procedure was used with strains NCTC 7011, BS 851 and BS 856 .

To estimate the mutants in cultures on solid media a loopful of an agar slant culture grown for $48 \mathrm{hr}$. at $30^{\circ}$ was suspended in a sterile physiological saline and plated as above.

Growth rates were determined from viable counts on populations allowed to grow for $22 \mathrm{hr}$. at $30^{\circ}$. As the growth constant we used the mean division rate $(r)$, calculated according to Monod (1949).

The biochemical tests were the same as in previous works (Kocur \& Martinec, 1959, 1963).

Each experiment described in this paper consists of several parallel observations from which the mean was calculated. With these parallels it was several times repeated. The results given involve the values of successful experiments which were analysed by conventional statistical methods, e.g. by the analysis of variance or by estimating the standard error $\left(s_{\bar{x}}^{-}\right)$. The value of $\delta$ was calculated according to the equation

$$
\delta=t \sqrt{\frac{2 \times \text { residual variance }}{m},}
$$

where $m$ is the number of members in the analysed group. 


\section{RESULTS}

Table 1 shows that after the second transfer the number of yellow-pigmented mutants reaches $8.73 \%$ of the Staphylococcus flavocyaneus population. After the eighth transfer the number of mutants reaches over $90 \%$.

Strains NCTC 7011, BS 851 and Bs 856 were similarly examined. After the tenth transfer there were on the average $8.35 \%$ of yellow-pigmented mutants in strain NCTC 7011 and $19 \cdot 1 \%$ in strain BS 851 . In BS 856 no yellow-pigmented mutants were found.

Table 1. Accumulation of yellow-pigmented mutants of Staphylococcus flavocyaneus during serial transfers in nutrient broth

Each population was initiated with $878 \pm\left(t s_{x}^{-}=65, P=0.05\right)$ cells of strain BS 852 and then after $48 \mathrm{hr}$. incubation at $30^{\circ} 0 \cdot 1 \mathrm{ml}$. of each population was transferred into fresh nutrient broth. The figures are the averages of six experiments.

$\begin{array}{cc}\begin{array}{c}\text { Average } \\ \text { percentage } \\ \text { of }\end{array} \\ \begin{array}{c}\text { No. of } \\ \text { transfers }\end{array} & \begin{array}{c}\text { mutants } \\ 2\end{array} \\ 4 & 30 \cdot 68 \\ 6 & 70 \cdot 16 \\ 8 & 91 \cdot 50 \\ 10 & 97 \cdot 33\end{array}$

Table 2. Mean division rates of strain $\mathrm{BS} 852$ and its yellowpigmented mutant in mixed and non-mixed populations

Mean division rates were estimated for the time interval of growth 0-22 hr. at $30^{\circ}$.

\begin{tabular}{|c|c|c|c|c|}
\hline & \multicolumn{2}{|c|}{ Mixed populations } & \multicolumn{2}{|c|}{ Non-mixed populations } \\
\hline & Parent & Mutant & Parent & Mutant \\
\hline Inocula & $212 \times 10^{4}$ & $356 \times 10^{2}$ & $266 \times 10^{4}$ & $503 \times 10^{4}$ \\
\hline $\begin{array}{l}\text { No. of cells per } 1 \mathrm{ml} . \pm t s_{x}^{-} \\
P=0.05\end{array}$ & $\pm 32 \times 10^{4}$ & $\pm 84 \times 10^{2}$ & $\pm 41 \times 10^{4}$ & $\pm 34 \times 10^{4}$ \\
\hline $\begin{array}{l}\text { Mean division rates per hour } \\
\text { (averages of six experiments) }\end{array}$ & $0 \cdot 242$ & 0.545 & 0.227 & $0 \cdot 274$ \\
\hline
\end{tabular}

The proportion of mutants in cultures grown on glucose yeast-extract agar slants and plates was on the average $0.21 \%$.

What causes the accumulation of the yellow-pigmented mutants? To answer this we determined the mean division rates of one of the mutant strains and of the parental strain. We used Bs 852/17, isolated from BS 852, as the yellow mutant strain. From Table 2 it is seen that the mean division rate of the mutant strain in non-mixed populations is only slightly higher than that of the parental strain. Nevertheless, the difference is significant $(\delta=\mathbf{0 . 0 3 0}$ calculated from the analysis of variance). However, the mean division rate of the mutant strain increased to approximately double in mixed populations. At the same time the mean division rate of the parental strain in the mixed populations was the same as that in the nonmixed populations (the difference was not significant statistically).

As to the phenotypic expression of the pigmentation by the parent and mutant 
strain, on some media the parent culture produces no violet diffusible pigment, or produces only very small amounts. The production of this pigment is slight or absent on nutrient agar, nutrient agar with starch, nutrient agar with gelatin and on yeast-extract agar with phenylalanine. On plates of these media the colonies of the parent culture appeared to be greenish yellow. On glucose yeast-extract agar with or without beef extract it produces both pigments. The mutant strain BS 852/17 produces yellow pigment on all these media.

Thirty yellow-pigmented mutants from Bs 852 were serially transferred in order to ascertain whether they lose the ability to produce the yellow pigment. No such loss was found even after fourteen transfers. We have been working with these mutants for 2 years (transfers into fresh media have been made at least once every two months) and we have not found any reversion to the violet-pigmented state or any other change in pigmentation.

Table 3. Taxonomic comparison of a yellow-pigmented mutant of strain Bs 852 with other strains of Micrococcus luteus

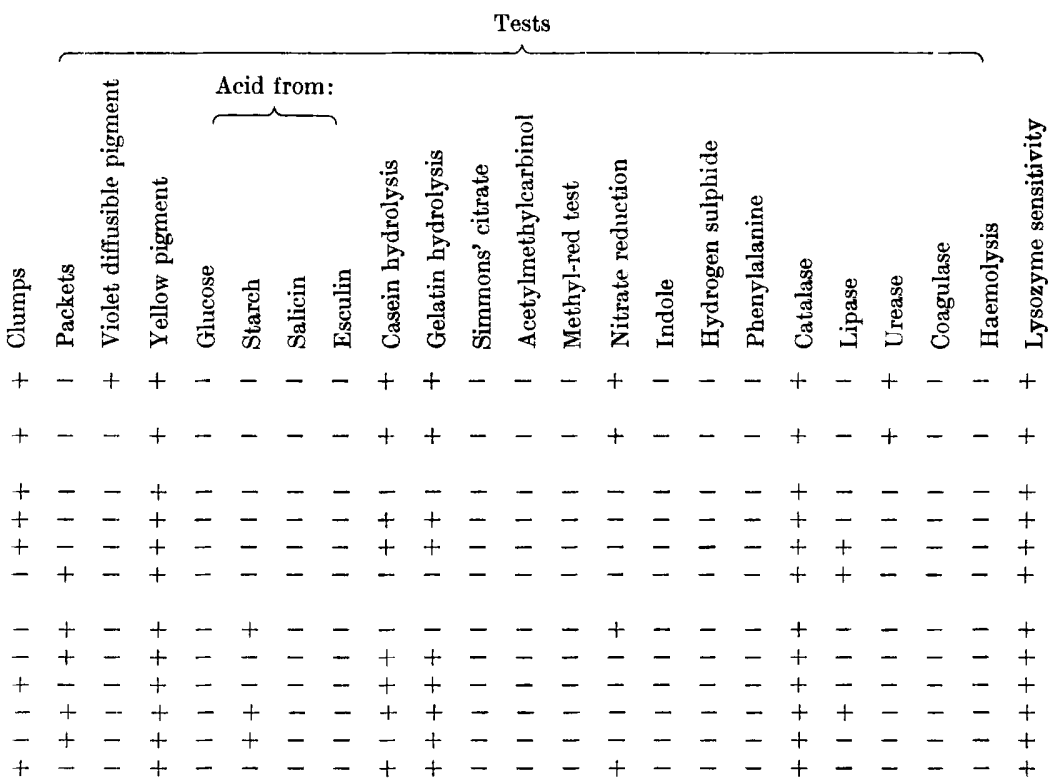

None of the thirty yellow-pigmented mutants differs except in pigmentation from the parental strain BS $\mathbf{8 5 2}$ in morphological, cultural and biochemical characters commonly used in taxonomy. One mutant strain, Bs 852/17, is compared with other yellow-pigmented cocci in Table $\mathbf{3}$. The names in Table $\mathbf{3}$ are commonly regarded as names of species, but we regard them all as strains of Micrococcus luteus (Kocur \& Martinec, to be published). The mutant strain only differs from the other strains in characteristics which (Kocur \& Martinec to be published) consider to be variable in $\boldsymbol{M}$. luteus. 


\section{DISCUSSION}

Metabolism of the organism can cause selection against the parent cells and favour other types (Braun et al. 1951; Braun, Firshein \& Whallon, 1957; Braun, 1958; Firshein \& Braun, 1958, 1960). Similarly in the broth cultures of Staphylococcus flavocyaneus the metabolism of parent cells may cause the selection of yellow-pigmented mutants so that the parent cells are outgrown by the mutant ones. Since the mean division rate of the mutant strain in unmixed populations was only slightly higher than that of the parent strain it follows that in broth cultures of $\boldsymbol{S}$. flavocyaneus conditions exist that favour the growth of yellow-pigmented mutants. These conditions created by the parent strain favour the survival of the mutants which outgrow the parent population.

The mutants did not revert to the violet pigmentation state. The production of yellow pigment by the mutants did not undergo phenotypic modification on different media, unlike the production of violet pigment by the parent culture, which was inhibited on some media. A convenient medium for the production of violet pigment seems to be glucose yeast-extract agar; on this and on other media, however, the yellow-pigmented mutants did not produce any violet pigment. This indicates that, as to pigmentations, the mutants differ genetically from the parent strain; they have lost by mutation the ability to produce the violet diffusible pigment. It was not possible, however, to measure the mutation rate by the methods of Stocker (1949) or Luria \& Delbrück (1943), because of the different growth rates and the absence of a selective medium for the yellow-pigmented mutants.

Yellow-pigmented mutants were not formed by Bs 856 under our conditions of cultivation. This strain is a rough $(\mathrm{R})$ form, whereas the strains BS 852, BS 851, and NCTC 7011 are smooth (S), and the environmental conditions for accumulating yellow-pigmented mutants may possibly be different.

Since the violet-pigmented cocci spontaneously throw off yellow variants that do not differ from other strains of Micrococcus luteus, except in a few characters that are variable in this species, we conclude that Staphylococcus flavocyaneus Knaysi is so closely related genetically to $M$. luteus (Schroeter) Cohn that it should be regarded as a strain of this species.

The authors wish to thank Miss Zdenka Vysloužilová for her excellent technical assistance in this work.

\section{REFERENCES}

Braun, W. (1958). Cell population dynamies and somatic change. J. cell. comp. Physiol. 52, 337.

Braun, W. Goodlow, R. J., Kraft, M., Altenbem, R. \& Mead, D. (1951). The effects of metabolites upon interactions between variants in mixed Brucella abortus populations. J. Bact. 62, 45.

Braun, W., Firshein, W. \& Whallon, J. (1957). Effects of desoxyribonucleic acid breakdown products on bacterial population changes and virulence. Science, 125, 445.

Firshein, W. \& Braun, W. (1958). On the nature of the selective effects of desoxyribonucleic acid digest upon pneumococci of different virulence. Proc. nat. Acad. Sci., Wash. 44, 918.

Firshein, W. \& Braun, W. (1960). Effects of enzymatic deoxyribonucleic acid digests on population changes and deoxyribonucleic acid synthesis of pneumococci. J.Bact. 79, 246. 
KNaysi, G. (1942). The demonstration of a nucleus in the cell of a staphylococcus. $J$. Bact. 43, 365.

Kocur, M. \& Martinec, T. (1959). Contribution to the taxonomic studies of Sarcina lutea Schroeter and Sarcina flava de Bary. Int. Bull. Bact. Nom. 9, 123.

Kocur, M. \& Martinec, T. (1963). The classification of some violet-pigmented micrococci. J. gen. Microbiol. 32, 185.

Luria, S. E. \& Delbrück, M. (1943). Mutations of bacteria from virus sensitivity to virus resistance. Genetics, $28,491$.

Monod, J. (1949). The growth of bacterial cultures. Annu. Rev. Microbiol. 3, 371.

STocker, B. A. D. (1949). Measurements of rate of mutation of flagellar antigenic phase in Salmonella typhimurium. J. Hyg., Camb. 47, 398. 